\title{
Measles vaccination coverage estimates from surveys, clinic records, and immune markers in oral fluid and blood: a population-based cross-sectional study
}

Kyla T Hayford ${ }^{1 *}$, Mohammed S Shomik ${ }^{2}$, Hassan M Al-Emran ${ }^{3}$, William J Moss ${ }^{4}$, David Bishai ${ }^{5}$ and Orin S Levine ${ }^{1}$

\begin{abstract}
Background: Recent outbreaks of measles and polio in low-income countries illustrate that conventional methods for estimating vaccination coverage do not adequately identify susceptible children. Immune markers of protection against vaccine-preventable diseases in oral fluid (OF) or blood may generate more accurate measures of effective vaccination history, but questions remain about whether antibody surveys are feasible and informative tools for monitoring immunization program performance compared to conventional vaccination coverage indicators. This study compares six indicators of measles vaccination status, including immune markers in oral fluid and blood, from children in rural Bangladesh and evaluates the implications of using each indicator to estimate measles vaccination coverage.

Methods: A cross-sectional population-based study of children ages 12-16 months in Mirzapur, Bangladesh, ascertained measles vaccination (MCV1) history from conventional indicators: maternal report, vaccination card records, 'card + history' and EPI clinic records. Oral fluid from all participants $(n=1226)$ and blood from a subset $(n=342)$ were tested for measles lgG antibodies as indicators of MCV1 history and compared to conventional MCV1 coverage indicators.
\end{abstract}

Results: Maternal report yielded the highest MCV1 coverage estimates (90.8\%), followed by EPI records (88.6\%), and card + history (84.2\%). Seroprotection against measles by OF (57.3\%) was significantly lower than other indicators, even after adjusting for incomplete seroconversion and assay performance (71.5\%). Among children with blood results, $88.6 \%$ were seroprotected, which was significantly higher than coverage by card + history and OF serostatus but consistent with coverage by maternal report and EPI records. Children with vaccination cards or EPI records were more likely to have a history of receiving MCV1 than those without cards or records. Despite similar MCV1 coverage estimates across most indicators, within-child agreement was poor for all indicators.

Conclusions: Measles IgG antibodies in OF was not a suitable immune marker for monitoring measles vaccination coverage in this setting. Because agreement between conventional MCV1 indicators was mediocre, immune marker surveillance with blood samples could be used to validate conventional MCV1 indicators and generate adjusted results that can be compared across indicators.

Keywords: Immunization, Vaccination, Immune marker, Surveillance, Measles, Oral fluid, Vaccination card, Maternal report, Bangladesh

\footnotetext{
* Correspondence: khayford@jhsph.edu

${ }^{1}$ Department of International Health, International Vaccine Access Center (IVAC), Johns Hopkins Bloomberg School of Public Health, 855 N. Wolfe Street, Suite 600, Baltimore, MD 21205, USA

Full list of author information is available at the end of the article
} 


\section{Background}

Surveillance of vaccination coverage is important for disease control, monitoring health system performance, and as a benchmark for progress toward Millennium Development Goal 4 on reducing child mortality [1-3]. However, conventional vaccination coverage estimates based on administrative records or household surveys are at risk of unintentional and intentional errors of omission, recording and recall [4-11]. Financial incentives for improving coverage can exacerbate these biases [11]. Administrative records from Expanded Programme on Immunisation (EPI) vaccination clinics generate vaccination coverage estimates in real-time with little added cost, but can suffer significant biases if reporting is incomplete or the eligible population size is not accurately measured [10,12-14]. Household surveys, such as the Demographic and Health Surveys (DHS) or Multiple Indicator Cluster Survey (MICS), generate three vaccination coverage indicators based on: 1) maternal report, 2) household-retained vaccination cards, or 3) a composite 'card + history' indicator, which uses vaccination card data or, if not available, maternal report. The 'card + history' indicator is regarded as the best available data source balancing accuracy with completeness but surveys are expensive and do not generate coverage estimates in real-time [6,7,15-17]. Consequently, comparisons of vaccination coverage using different indicators are often invalid [4-6,10-12].

Recent outbreaks of measles in low-income countries highlight that conventional vaccination coverage indicators do not adequately identify susceptible children. There is increasing interest in immune marker surveillance to monitor immunization programs and estimate population immunity attributable to vaccination [18-20]. However, immune marker surveillance with blood or oral fluid (OF) samples pose other errors and biases related to sample collection, storage, testing and interpretation of results as well as financial and logistical challenges in the field [21]. In this study, we compared six indicators of measles vaccination status, including immune markers of measles immunity in oral fluid and blood, from young children in rural Bangladesh and evaluated the implications of using each indicator to estimate measles vaccination (MCV1) coverage.

\section{Methods}

The study was conducted among children aged 1216 months residing in the Mirzapur Demographic Surveillance System (DSS), a rural area $60 \mathrm{~km}$ north of Dhaka, Bangladesh. The DSS is comprised of $\sim 240,000$ individuals living in 58,300 households visited every 4 months to update births, deaths and migrations. A stratified random sample of 1450 children was selected from the eight unions (administrative unit similar to a county) of the DSS with probability of proportional to eligible population size in each union. Ethics committees at the International Centre for Diarrhoeal Disease Research, Bangladesh (icddr,b) and the Johns Hopkins Bloomberg School of Public Health approved the study and written permission from the participants' guardian was obtained prior to enrollment.

Data collection took place in three stages: a caregiver survey and oral fluid collection in the household, review of EPI clinic records, and blood collection from a subset of children in four of the eight unions. A wealth index was generated using principal component analysis based on household assets [22]. Record books from EPI clinics were obtained and records were matched to enrolled children based on EPI registration IDs from their vaccination cards or by matching at least three variables: child name, birth date ( \pm 3 days), father's name or grandfather's name.

OF samples were obtained by rubbing a foam swab (Oracol; Malvern Medical Developments, Worcester, UK) along the child's gumline for 1-1.5 minutes as described elsewhere [23]. OF samples were transported in cold boxes within 4 hours to the local laboratory where $1 \mathrm{~mL}$ of transport buffer was added, centrifuged at $2000 \mathrm{rpm}$ for five minutes, and the supernatant was stored at $-20^{\circ} \mathrm{C}$ until testing. OF samples were tested for antibodies to measles virus using a measles virus-specific IgG capture enzyme immunoassay (ELISA) (Microimmune Ltd, Middlesesex, UK), which was validated for use with OF samples with a reported sensitivity of $93 \%$ and specificity of $98 \%$ compared to serum [24]. OF samples were categorized as positive, equivocal or negative; equivocal samples were retested and, if equivocal again, were considered positive in binary analyses. To identify poor quality OF samples, specimens testing negative for measles IgG were tested for total IgG antibodies by ELISA (Bethyl Laboratories, Montgomery, TX) and excluded from the analysis if less than $125 \mathrm{ng} / \mathrm{mL}$.

Peripheral blood samples were collected by trained phlebotomists from all enrolled children with parental permission within one month of OF collection in four of eight unions. Serum samples were extracted and stored at $-20^{\circ} \mathrm{C}$ until tested for measles IgG antibodies (Enzygnost ELISA, Siemens, Germany) with a reported sensitivity and specificity of $99.6 \%$ and $100 \%$, respectively [25]. Serum samples were categorized as negative, equivocal or positive as recommended by the manufacturer. Serum samples testing equivocal were re-tested and, if equivocal again, were categorized as positive.

Up to six indicators of MCV1 history were ascertained for each child: 1) maternal report of MCV1 based on recall questions modified from the DHS [26]; 2) card record of MCV1 based on evidence and dates of MCV1 receipt on the child's household retained vaccination card, if available; 3) 'card + history'; 4) EPI record of MCV1 based on dates and evidence of MCV1 abstracted from EPI clinic 
record books; 5) protective levels of measles IgG antibodies in OF; and 6) protective levels of measles IgG antibodies in blood. MCV1 coverage by OF was adjusted for assay sensitivity and specificity using following equation: $\mathrm{P}_{\text {adjusted }}=\left(\mathrm{P}_{\text {observed }}-(1\right.$-specificity $\left.)\right) /(1-[(1$-specificity $)+$ (1-sensitivity)]). Seroprotection in OF and blood was assumed to be vaccine induced because maternal antibodies should have been non-detectable is this age group and the last measles outbreak in the district occurred more than a year before study participants were born according to the WHO measles surveillance laboratory in Dhaka, Bangladesh [27,28]. Study participants were not age eligible for the 2010 MCV campaign.

Categorical variables were compared using chi-square tests or Fisher's exact test and continuous variables with non-normal distributions were compared using the Wilcoxon rank sum test. MCV1 coverage estimates were generated with exact binomial confidence intervals and compared using McNemar's test for paired samples. Bivariate logistic and log binomial models were used to calculate odds ratios and prevalence ratios, respectively. Sensitivity, specificity and kappa were calculated to evaluate within-child agreement of indicators. Analysis was conducted in Stata 11 (StataCorp LP, College Park, TX, USA).

\section{Results}

1450 children were randomly selected from the DSS database, of whom 1389 were living in the DSS area at the time of the study. Of these children, 1260 (89.8\%) were enrolled in the study from September 2010 to January 2011 (Figure 1, Additional file 1: Table S1). The parent of one child refused OF collection and 33 OF samples were excluded for poor quality (total IgG antibodies $<125 \mathrm{ng} / \mathrm{mL}$ ), resulting in 1226 children in the primary analysis. Vaccination records from EPI record books were found for 913 (72.5\%) children, of whom 891 had an adequate OF sample. Blood was collected from 342 children with a $7.7 \%$ refusal rate. Of children with blood sample results, 311 (91\%) had adequate OF samples and 268 (78\%) had EPI clinic records.

No differences in the age, sex, asset quintile, household size, or education level of the father was observed for enrolled versus non-enrolled children, but mothers with more education (unadjusted OR 0.94 for each additional year; 95\% CI: 0.89, 0.99), working mothers (OR 0.38; CI: 0.21, 0.68), and families that had moved at least once since 2005 (OR 0.47; CI: 0.32, 0.68) were significantly less likely to be enrolled.

\section{Factors associated with MCV1 history}

The mean age of enrolled children was 14.5 months with an equal proportion of males and females (Table 1). Using the card + history indicator as the best available data source, MCV1 coverage did not differ by age or sex, but children taken by family members other than the mother for vaccination were less likely to have received MCV1.

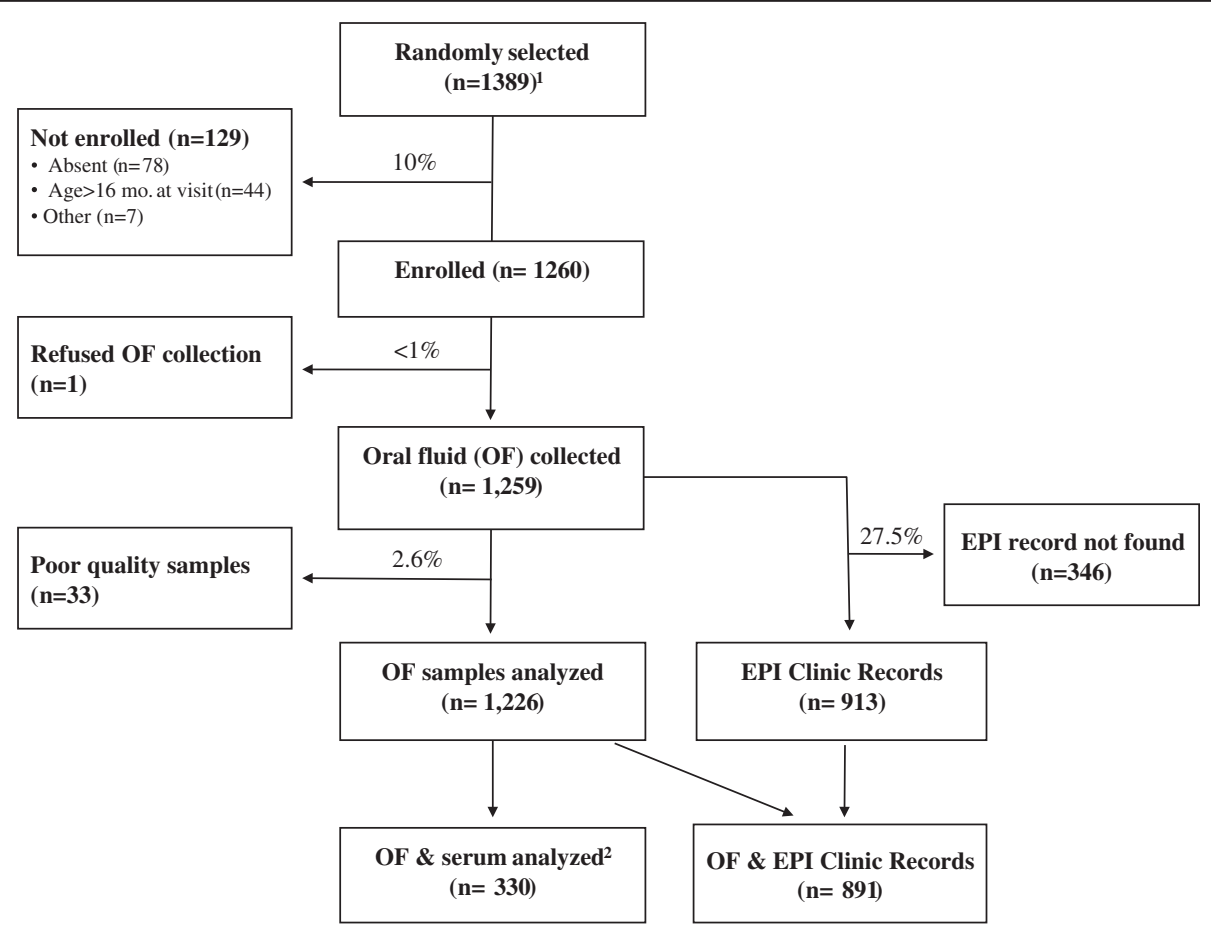

Figure 1 Enrollment chart. ${ }^{1} 61$ children selected from the database were not included because they migrated out of DSS before initiation of study. ${ }^{2}$ Blood samples were collected and analyzed from 342 children. Paired OF and blood samples were available for 330 children. 
Table 1 Characteristics of enrolled children and factors associated with receipt of MCV1

\begin{tabular}{|c|c|c|c|c|c|}
\hline & & & Card + His & indicator & \\
\hline & Frequency & $\begin{array}{c}\text { Overall } \\
\%\end{array}$ & $\begin{array}{c}\text { Vaccinated } \\
\text { (1033 children) }\end{array}$ & $\begin{array}{l}\text { Unvaccinated } \\
\text { (193 children) }\end{array}$ & p-value ${ }^{1}$ \\
\hline Age at enrollment, mean (SD) & $14.5(1.4)$ & & $14.5(1.5)$ & $14.5(1.4)$ & 0.930 \\
\hline Male & 628 & 51.2 & $534(51.7)$ & $94(48.7)$ & 0.446 \\
\hline Father's education, median [IQR] (years) & $7[5,9]$ & & $7[5,10]$ & $5[0,9]$ & $<0.001$ \\
\hline Mother's education, median [IQR](years) & $7[4,9]$ & & $7[5,9]$ & $5[2,8]$ & $<0.001$ \\
\hline Mother had job in last 3 years & 154 & 12.6 & $116(11.2)$ & $38(19.7)$ & 0.001 \\
\hline Asset quintile $(2010)^{2}$ & & & & & \\
\hline 1 - poorest & 246 & 20.1 & $192(18.6)$ & $54(28.0)$ & 0.001 \\
\hline 2 & 250 & 20.4 & $202(19.6)$ & $48(24.9)$ & \\
\hline 3 & 246 & 20.1 & $208(20.1)$ & $38(19.7)$ & \\
\hline 4 & 243 & 19.8 & $215(20.8)$ & $28(14.5)$ & \\
\hline 5 - wealthiest & 241 & 19.7 & $216(20.9)$ & $25(13.0)$ & \\
\hline Retained child's vaccination card & 1019 & 83.1 & $872(84.4)$ & $147(76.1)$ & 0.005 \\
\hline Mother responsible for bringing child for vaccinations & 1060 & 86.5 & $903(87.4)$ & $159(79.9)$ & 0.006 \\
\hline Location of child's delivery ${ }^{3}$ & & & & & \\
\hline Hospital or clinic & 460 & 37.5 & $402(38.9)$ & $58(30.0)$ & 0.034 \\
\hline House (own, family or friend's home) & 752 & 61.3 & $621(60.1)$ & $131(67.9)$ & \\
\hline Person assisting with child's delivery ${ }^{3}$ & & & & & \\
\hline Qualified doctor & 283 & 23.1 & $253(24.5)$ & $30(15.4)$ & 0.029 \\
\hline Skilled birth attendant & 209 & 17.0 & $178(17.2)$ & $31(16.1)$ & \\
\hline Traditional birth attendant & 73 & 54.9 & $554(53.6)$ & $119(61.7)$ & \\
\hline Other (mother, informal health worker, alone) & 47 & 3.8 & $38(3.7)$ & $9(4.7)$ & \\
\hline Antenatal care visits $^{3}$ & & & & & \\
\hline None & 320 & 26.1 & $251(24.3)$ & $69(35.8)$ & $<0.001$ \\
\hline 1 & 246 & 20.1 & $203(19.6)$ & $43(22.3)$ & \\
\hline 2 or more & 645 & 52.6 & $568(55.0)$ & $77(39.9)$ & \\
\hline Postnatal care visits ${ }^{3}$ & & & & & \\
\hline None & 645 & 52.6 & $525(50.8)$ & $120(62.2)$ & 0.007 \\
\hline 1 & 412 & 33.6 & $364(35.2)$ & $48(24.9)$ & \\
\hline 2 or more & 155 & 12.6 & $134(13.0)$ & $21(10.9)$ & \\
\hline
\end{tabular}

${ }^{1}$ Chi-square or Fisher's exact test for categorical variables. Wilcoxon rank sum test for non-normal continuous variables.

${ }^{2}$ Asset quintiles calculated using principal component analysis from household asset data.

${ }^{3}$ Data on child's delivery $(n=14)$, antenatal care $(n=15)$ and postnatal care $(n=14)$ not available for small number of children.

Higher socioeconomic status (SES) based on asset quintiles was associated with receipt of MCV1. Children vaccinated against measles were more likely to be born in a hospital or clinic, have a skilled birth attendant or doctor assist in their delivery, and have more antenatal and postnatal care visits than unvaccinated children (Table 1).

\section{MCV1 Coverage by indicator}

Maternal report yielded the highest coverage estimates (90.8\% CI: 89.0, 92.3), followed by EPI records (88.6\%; CI: 86.3, 90.6), card records among card holders (85.6\%; CI: 83.3, 87.7), and card + history (84.2\%; CI: 82.1, 86.2).
Coverage of protective levels of measles IgG antibodies in OF $(57.3 \%$; CI: $54.5,60.1)$ was significantly lower than conventional indicators (Figure 2). Two adjustments were made to the raw OF prevalence to account for differences between vaccination history and immunologic status. Assuming 85\% seroconversion among children ages $9-10$ months, MCV1 coverage by OF was $67.4 \%$ (i.e. $57.3 / 0.85=67.4 \%$ ) [29]. A subsequent adjustment accounting for the reported sensitivity and specificity of the assay generated a final adjusted MCV1 coverage estimate of $71.5 \%$ by OF, which remained statistically significantly lower than coverage based on all conventional 


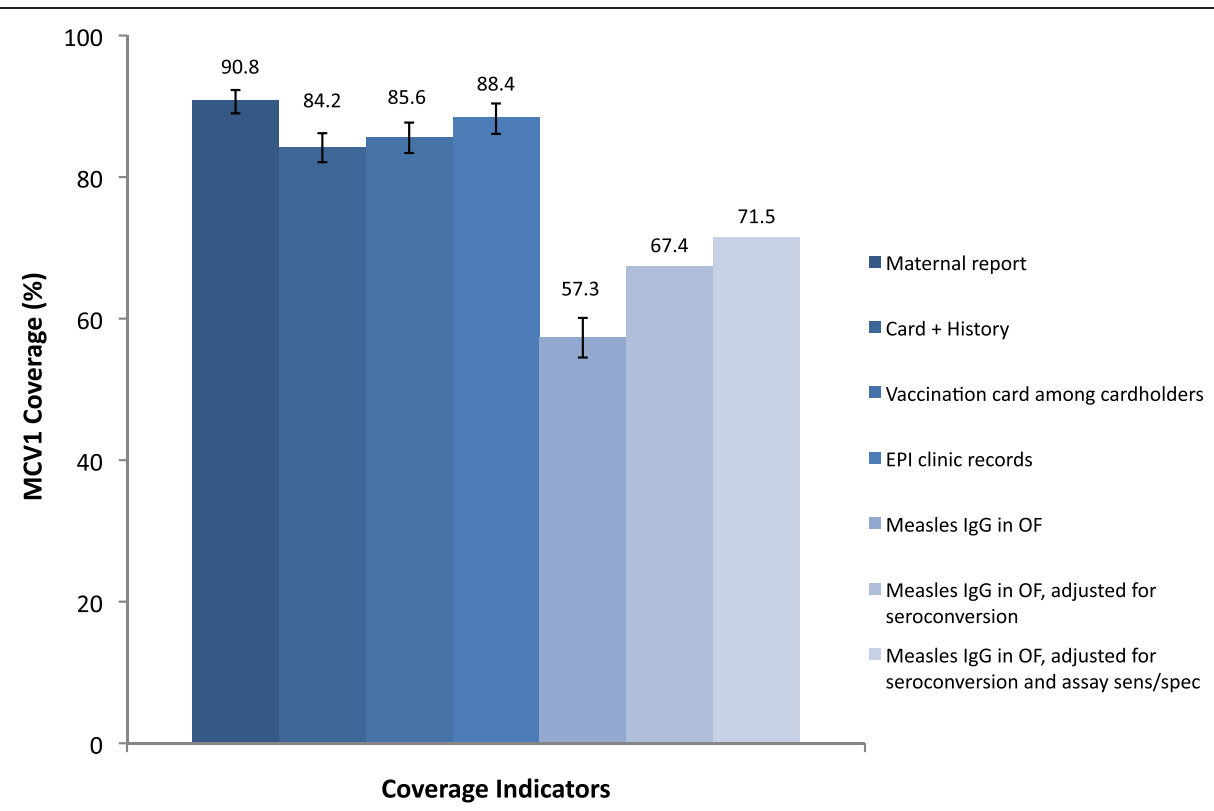

Figure 2 Overall MCV1 Coverage by Indicator. Note: MCV1 coverage based on all available data and sample size varies by indicator. 'Oral fluid adjusted for seroconversion' assumes $85 \%$ of vaccinated children seroconverted. 'Oral fluid, adjusted for seroconversion and assay sensitivity/ specificity' assumes $85 \%$ seroconversion and adjusts for the sensitivity (93\%) and specificity (98\%) of the assays, as reported by the manufacturer.

MCV1 indicators (McNemar's test, $\mathrm{p}<0.001$ for all pairwise tests) (Figure 3). For the 342 children with blood results, $83.0 \%$ had protective levels of measles IgG antibodies, $11.2 \%$ were susceptible and $5.8 \%$ had equivocal results, which were categorized as positive in binary analyses for an overall prevalence of $88.6 \%$ (CI: 85.2, 92.0). For children with both blood and OF samples, MCV1 coverage by blood serostatus was significantly higher than by card + history $(84.5 \%$, McNemar's test $\mathrm{p}=0.038$ ) but non-significant differences were detected

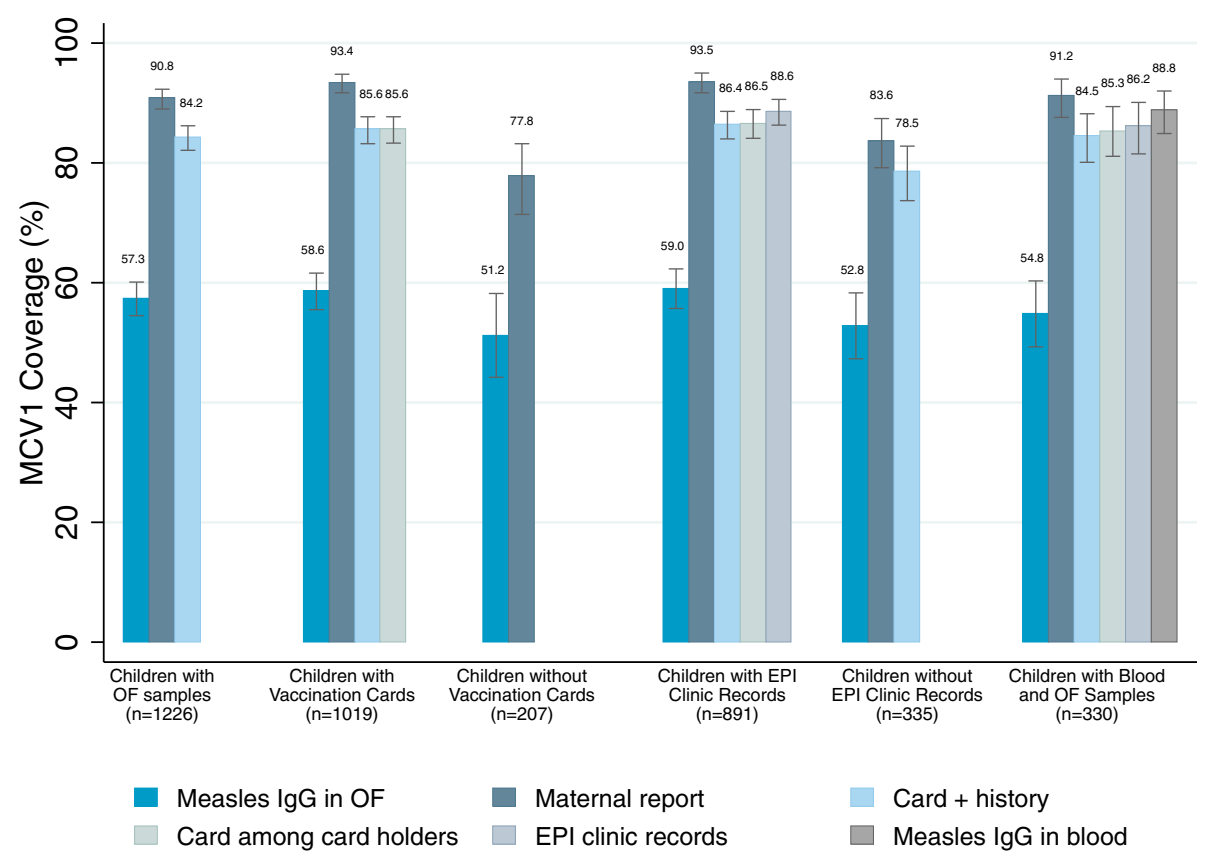

Figure 3 Measles vaccination coverage by indicator among subgroups of children with complete data. Note: Coverage from EPI records is not representative of the population because $\sim 27 \%$ of records could not be located. Children with blood samples represent a subset of study population ( $n=330$ except for EPI clinic records where $n=268$ ). 
for maternal report $(90.9 \%, \mathrm{p}=0.10)$ and EPI records $(86.0 \%, \mathrm{p}=0.16)$.

To account for missing data for several indicators, MCV1 coverage indicators were compared within subgroups of children who had complete data (Figure 3). Within each subgroup, MCV1 coverage based on OF was significantly lower than all other coverage indicators $(\mathrm{p}<$ 0.001 for all pairwise tests). MCV1 coverage by maternal report was consistently higher than other indicators $(\mathrm{p}<$ 0.001 for all pairwise tests) except among those with both blood and OF samples. To capture systematic differences between indicators, prevalence ratios comparing MCV1 coverage for each pair of indicators were generated to show the observed over- or under-estimation in MCV1 coverage estimates (Table 2).

$83 \%$ of parents showed their child's vaccination card at the interview, of which $85.6 \%$ (CI: 83.4, 87.7) had evidence of MCV1 on the card (Figure 3). Children with vaccination cards were significantly more likely to have received MCV1 than children without cards by all conventional indicators and blood serostatus (maternal report: $93.4 \%$ (card) vs. $77.8 \%$ (no card), $\mathrm{p}<0.001$; EPI records: $86.9 \%$ vs. $100 \%, p=0.006$; OF: $58.6 \%$ vs. $51.2 \%$, $\mathrm{p}=0.054$; blood: $90.5 \%$ vs. $77.8 \%, \mathrm{p}=0.02$ ). EPI records were located for $72.5 \%$ of enrolled children. Children with available EPI records were more likely to be younger, have lower asset scores, not moved since the child was born, and retained the child's vaccination card than children whose records were not located (Table 3). Children with EPI records had significantly higher MCV1 coverage by maternal report $(93.5 \%$ with EPI records vs. 83.6\% without EPI records, $\mathrm{p}<0.001)$, card + history ( $86.4 \%$ vs. $78.5 \%, \mathrm{p}=0.001)$, and blood serostatus $(90.7 \%$ vs. $80.6 \%, \mathrm{p}=0.041)$.
Higher asset quintiles were associated with higher MCV1 coverage for all indicators except card records and EPI records with the most substantial gap between the bottom $20 \%$ and top $80 \%$ of asset scores (Table 4). The relative odds of MCV1 history by maternal report was 2.41 times greater than for children in the top $80 \%$ of asset scores (CI: 1.59, 3.65). Restricting analyses to children with blood data attenuated differences across asset quintiles for all indicators except coverage based on blood serostatus. Children in the highest $80 \%$ of asset scores had significantly higher odds of having protective levels of measles IgG antibodies in blood than children in the lowest $20 \%$ (OR 2.48; CI: 1.23, 5.01), whereas there was no difference for maternal report (OR 1.77; CI: 0.79, 3.94), card + history (OR 1.31; CI: 0.67, 2.57), or EPI records (OR 0.90; CI: 0.40, 1.99) (Table 4). Significant differences in measles serostatus remained between the poorest quintile and other quintiles after adjusting for MCV1 history (card + history) (OR 2.74; CI: $1.10,6.90)$.

\section{Agreement \& performance of MCV1 indicators at individual level}

We also assessed within-child agreement for each data source. MCV1 history by OF had poor sensitivity (62.3\%; CI: $59.3,65.3)$ and specificity $(69.4 \%$; CI: 62.4, 75.8) compared to card + history, which was considered the gold standard. Maternal report had high sensitivity (99.4\%; CI: 98.7, 99.8) but poor specificity (55.4\%; CI: 48.1, 62.6) (Additional file 1: Table S2). To assess agreement between maternal report and card records, the analysis was restricted to children with vaccination cards. $99 \%$ of mothers with card-confirmed MCV1 reported MCV1 history. For those without card-confirmed MCV1, 43\% still reported MCV1 history, resulting in 91\% agreement and a kappa of

Table 2 Prevalence ratios for MCV1 coverage to adjust for differences between indicators: an approach to comparing vaccination coverage indicators

\begin{tabular}{lccc}
\hline $\begin{array}{l}\text { Comparison indicator } \\
\text { Reference indicator }\end{array}$ & \multicolumn{3}{c}{ Prevalence ratio [95\% Cl] } \\
\cline { 2 - 4 } Serum & Maternal report & Card + History & EPI records \\
& 1.026 & $\mathbf{0 . 9 5 4}$ & 0.952 \\
& {$[0.998,1.056]$} & {$[\mathbf{0 . 9 1 4 , 0 . 9 9 6 ]}$} & $\mathrm{n}=278$ \\
EPI records & $\mathrm{n}=342$ & $\mathrm{n}=342$ & - \\
& $\mathbf{1 . 0 5 7}$ & 0.976 & - \\
& {$[\mathbf{1 . 0 2 4} \mathbf{1 . 0 8 8}]$} & {$[0.940,1.013]$} & $\mathrm{n}=891$ \\
Card + History & $\mathrm{n}=891$ & - & \\
& $\mathbf{1 . 0 7 7}$ & &
\end{tabular}

Note: Estimates are prevalence ratios [95\% confidence intervals] to estimate the degree of over- or underestimation for each method used. The prevalence ratio based on comparison indicator (row) divided by reference indicator (column). E.g. the maternal report indicator generates an MCV1 coverage estimate $7.7 \%$ higher (PrR: $1.077=0.9078 / 0.8426)$ than coverage estimated by card + history. Bold indicates statistical significance with a $5 \%$ two-sided alpha. Results from this study are presented as an example of a tool governments or EPI programs could develop to compare vaccination coverage estimates from different indicators. 
Table 3 Characteristics of children with and without EPI clinic records

\begin{tabular}{|c|c|c|c|c|c|}
\hline & \multicolumn{2}{|c|}{ EPI records found $(n=913)$} & \multicolumn{2}{|c|}{ EPI records not found $(n=346)$} & \multirow[b]{2}{*}{ p-value ${ }^{1}$} \\
\hline & Frequency & $\%$ & Frequency & $\%$ & \\
\hline Age at enrollment (months), mean (SD) & $13.8(1.4)$ & & $14.0(1.4)$ & & 0.026 \\
\hline Male & 456 & 50.0 & 186 & 53.8 & 0.227 \\
\hline Father's Education, in years, median (IQR) & $6[4,9]$ & & $8[5,10]$ & & $<0.001$ \\
\hline Moved since child's birth & 327 & 35.8 & 163 & 47.1 & $<0.001$ \\
\hline \multicolumn{6}{|l|}{ Asset quintile } \\
\hline 1 (poorest) & 198 & 21.7 & 55 & 15.9 & 0.001 \\
\hline 2 & 191 & 20.9 & 61 & 17.6 & \\
\hline 3 & 177 & 19.4 & 75 & 21.7 & \\
\hline 4 & 189 & 20.7 & 62 & 17.9 & \\
\hline 5 (wealthiest) & 158 & 17.3 & 93 & 26.9 & \\
\hline Retained vaccination card & 800 & 87.6 & 246 & 71.1 & $<0.001$ \\
\hline History of MCV1 by maternal report & 853 & 93.4 & 287 & 83.0 & $<0.001$ \\
\hline History of MCV1 by card + history & 790 & 87 & 270 & 78 & $<0.001$ \\
\hline Fully vaccinated, by card + history & 657 & 72.0 & 187 & 54.0 & $<0.001$ \\
\hline \multicolumn{6}{|l|}{ Union } \\
\hline Ajgana & 139 & 15.2 & 67 & 19.4 & $<0.001$ \\
\hline Bahuria & 146 & 16.0 & 25 & 7.2 & \\
\hline Banail & 104 & 11.4 & 14 & 4.0 & \\
\hline Bhatgram & 108 & 11.8 & 20 & 5.8 & \\
\hline Gorai $^{2}$ & 98 & 10.7 & 114 & 33.0 & \\
\hline Jamurki & 101 & 11.1 & 45 & 13.0 & \\
\hline Uarsi & 116 & 12.7 & 12 & 3.5 & \\
\hline Mirzapur & 101 & 11.1 & 49 & 14.1 & \\
\hline
\end{tabular}

${ }^{1}$ Chi square test for categorical variables. Wilcoxon rank sum test for non-normally distributed continuous variables.

${ }^{2}$ Record books from two EPI clinics in Gorai were not available.

0.54 (95\% CI: 0.46, 0.62) between vaccination cards and maternal report. 150 children had vaccination cards but lacked evidence of MCV1 receipt on their card. Among the $150,105(70 \%)$ had a date of MCV1 receipt in the EPI record books, 86 (57\%) mothers reported the child received MCV1, and 67 (45\%) had both. 43 of the 150 children had blood results, of which 25 (58\%) were seropositive and only 18 (42\%) were seronegative as expected from their vaccination card.

Similarities in coverage at the population level often masked discordance between indicators. Among children with both vaccination cards and EPI records, MCV1 coverage was the same by EPI record or vaccination card but within-child agreement was only $73.8 \%$ (CI: 70.6, 76.8) because an equal number of children were positive by one source and negative by the other (Table 5 ).

\section{Discussion}

Using surveys, clinic records and immunologic markers, up to six measures of MCV1 coverage were generated for a representative sample of children in rural Bangladesh.
No prior study has compared as many indicators including immune markers from a single cohort of children. Apart from OF results, vaccination coverage estimates did not vary dramatically across data sources or indicators. MCV1 coverage from reported or recorded data ranged from $84 \%$ to $91 \%$, with a best estimate of $84 \%$ based on the 'gold standard' card + history indicator. A 2010 Bangladesh government survey using the card + history indicator found $84 \%$ MCV1 coverage for the district, a catchment area slightly larger than the DSS [30].

Immune marker surveillance with OF was not an accurate indicator of vaccination history compared to all other indicators. The proportion of children with detectable antibodies in OF was significantly lower than expected by reported or recorded MCV1 coverage even after adjusting for incomplete seroconversion and assay performance [29]. In the subset of children with blood samples, poor agreement between blood and OF results reinforced that OF testing was not an accurate immune marker and is not recommended for use in immunization surveillance unless the validity and reliability of OF testing can be improved [31]. 
Table 4 Association between indicators of MCV1 coverage and socioeconomic status

\begin{tabular}{|c|c|c|c|c|c|c|c|}
\hline \multicolumn{8}{|c|}{ All children in study $(n=1226)$} \\
\hline & \multicolumn{5}{|c|}{ Asset quintile } & \multirow[t]{2}{*}{ Total } & \multirow[t]{2}{*}{ p-value ${ }^{2}$} \\
\hline & 1 - poorest & 2 & 3 & 4 & 5 - wealthiest & & \\
\hline \multirow[t]{2}{*}{ Maternal recall $(n=1226)^{1}$} & 83.7 & 88.0 & 93.1 & 95.1 & 94.2 & 90.8 & \\
\hline & $(78.4,88.1)$ & $(83.3,91.8)$ & $(89.2,95.9)$ & $(91.5,97.4)$ & $(90.4,96.8)$ & $(89.0,92.3)$ & $<0.001$ \\
\hline \multirow[t]{2}{*}{ Card + History $(n=1226)^{1}$} & 78.0 & 80.8 & 84.6 & 88.5 & 89.6 & 84.2 & 0.003 \\
\hline & $(72.3,83.1)$ & $(75.4,85.5)$ & $(79.4,88.8)$ & $(83.8,92.2)$ & $(85.1,93.2)$ & $(82.1,86.2)$ & \\
\hline \multirow[t]{2}{*}{ Card record $(n=1046)$} & 82.8 & 84.7 & 84.8 & 86.7 & 89.4 & 85.7 & \\
\hline & $(77.0,87.6)$ & $(79.1,89.3)$ & $(79.4,89.2)$ & $(81.2,91.1)$ & $(84.4,93.2)$ & $(83.4,87.7)$ & 0.135 \\
\hline \multirow[t]{2}{*}{ Oral fluid $(n=1226)^{1}$} & 49.2 & 55.2 & 60.6 & 61.7 & 60.2 & 57.3 & \\
\hline & $(42.8,55.6)$ & $(48.8,61.5)$ & $(54.2,66.7)$ & $(55.3,67.9)$ & $(53.7,66.4)$ & $(54.5,60.1)$ & 0.004 \\
\hline \multirow[t]{2}{*}{ EPI records $(n=913)$} & 87.9 & 89.0 & 88.7 & 91.0 & 84.8 & 88.4 & \\
\hline & $(82.5,92.1)$ & $(83.7,93.1)$ & $(83.1,93.0)$ & $(86.0,94.6)$ & $(78.2,90.0)$ & $(86.1,90.4)$ & 0.25 \\
\hline$\%$ of EPI records found & $78 \%$ & $76 \%$ & $70 \%$ & $75 \%$ & $63 \%$ & $73 \%$ & \\
\hline \multicolumn{8}{|c|}{ Children with blood samples $(n=342)$} \\
\hline & 1 - poorest & 2 & 3 & 4 & 5 - wealthiest & Total & p-value ${ }^{2}$ \\
\hline \multirow[t]{2}{*}{ Maternal recall $(n=342)$} & 86.8 & 87.3 & 92.7 & 93.8 & 94.7 & 90.9 & \\
\hline & $(77.1,93.5)$ & $(77.3,94.0)$ & $(82.4,98.0)$ & $(84.8,98.3)$ & $(87.1,98.5)$ & $(87.4,93.8)$ & 0.163 \\
\hline \multirow[t]{2}{*}{ Card + History $(n=342)$} & 81.6 & 74.6 & 85.5 & 87.5 & 93.4 & 84.5 & \\
\hline & $(71.0,89.5)$ & $(62.9,84.2)$ & $(73.3,93.5)$ & $(76.8,94.4)$ & $(85.3,97.8)$ & $(80.2,88.2)$ & 0.425 \\
\hline \multirow[t]{2}{*}{ Card record $(n=294)^{1}$} & 82.1 & 78.3 & 86.3 & 88.0 & 92.4 & 85.4 & \\
\hline & $(70.8,90.4)$ & $(65.8,87.9)$ & $(73.7,94.3)$ & $(75.7,95.5)$ & $(83.2,97.5)$ & $(80.8,89.2)$ & 0.388 \\
\hline \multirow[t]{2}{*}{ Oral fluid $(n=330)^{1}$} & 50.7 & 52.8 & 54.7 & 61.7 & 55.4 & 54.8 & \\
\hline & $(38.7,62.6)$ & $(40.6,64.9)$ & $(40.4,68.4)$ & $(48.2,73.9)$ & $(43.4,67.0)$ & $(49.3,60.3)$ & 0.418 \\
\hline \multirow[t]{2}{*}{ Serum $(n=342)$} & 80.2 & 90.1 & 89.1 & 90.6 & 93.4 & 88.6 & \\
\hline & $(69.5,88.5)$ & $(80.7,95.9)$ & $(77.8,95.9)$ & $(85.3,98.9)$ & $(85.3,97.8)$ & $(85.2,92.0)$ & 0.011 \\
\hline \multirow[t]{2}{*}{ EPI records $(n=278)^{1}$} & 87 & 89.6 & 91.3 & 88.4 & 73.6 & 86 & \\
\hline & $(76.7,93.8)$ & $(78.8,96.1)$ & $(79.2,97.6)$ & $(76.6,95.6)$ & $(59.7,84.7)$ & $(81.3,89.8)$ & 0.786 \\
\hline$\%$ of EPI records found & $91 \%$ & $82 \%$ & $84 \%$ & $82 \%$ & $70 \%$ & $81 \%$ & \\
\hline
\end{tabular}

Our results highlighted that mothers who received antenatal, delivery and postnatal care in the formal health system were more likely to have MCV1 vaccinated children. Unlike some other studies from South Asia, boys were not more likely than girls to be vaccinated with MCV1 $[32,33]$. Unvaccinated children were more likely to live in households with relatively lower SES and education. After accounting for reported vaccination history, children in

Table 5 Discordance between MCV1 history in EPI records $\&$ vaccination cards

\begin{tabular}{llcc}
\hline & \multicolumn{2}{c}{ Vaccination card } \\
\cline { 3 - 4 } & MCV1 (+) & MCV1 (-) \\
\hline EPI & MCV1 (+) & 694 & 105 \\
records & MCV1 (-) & 105 & 1 \\
\hline
\end{tabular}

Note: Includes only children with vaccination card records and EPI clinic records $(n=800)$. the poorest $20 \%$ were significantly more likely to be measles susceptible than those in the upper $80 \%$, warranting further investigation into biological or systems-level causes of susceptibility among the very poor $[34,35]$.

Many studies have shown that reported and recorded vaccination history data are fraught with biases and errors in recall and omission [4,6,8,11,16,36,37]. Studies in Bangladesh [38], Costa Rica [9], and England [39] found that maternal report inflated coverage estimates but our results illustrated that maternal report or card + history indicators performed well compared to serological and government estimates of MCV1 coverage. Without a gold standard of vaccination history, it was not possible to assess if maternal report overestimated coverage. What may be more important is to generate vaccination coverage estimates that can be compared across indicators. Currently policymakers frequently compare vaccination coverage 
estimates generated from different indicators and data sources, resulting in an 'apples to oranges' comparison. Table 2 presents an example of how adjustment factors could be generated for each pair of coverage indicators to evaluate the degree of over- or underestimation with each indicator. Because the indicators used in this study were generated in the same way as national coverage estimates from administrative records, coverage evaluation surveys and DHS surveys, a similar table of adjustment factors could be generated from a small representative sample of children in a country and then used to adjust national coverage estimates to make them more comparable between data sources.

Our results called into question whether vaccination card records should be considered the best available information for a child's vaccination status because missing vaccination records on the card could be verified with other data sources. DHS and MICS surveys seek maternal report of vaccination history only when the child's vaccination card is not available. Future studies should explore if vaccination history by maternal report should also be used for children who have cards but lack evidence of the vaccination on their card.

Because children with cards were more likely to be vaccinated and their mothers were more likely to correctly recall MCV1 status, survey with higher card retention rates may have less error in coverage estimates. Consequently, we recommend that the card retention rates should always be presented as an indicator of data quality when the card + history indicator is used.

Our results should be interpreted in light of the limitations of the data. Without a vaccination registry, there was no gold standard measures of vaccination history or measles immunity [40]. We used strict criteria for matching children with their EPI clinic records to avoid matching errors, but this may have underestimated the number of children with EPI records and induced a bias that overestimated MCV1 coverage by EPI record. Results from OF testing generated significant concern about its validity for immunization surveillance. Several other studies have shown good sensitivity and specificity for the assay, suggesting that the problems in this study could be ameliorated [41-43].

\section{Conclusions}

This study illustrates that a simple comparison of data can have broad implications for immunization surveillance. Surveys using the card + history or maternal report indicators combine feasibility with accuracy in this setting and continued use is recommended to monitor secular trends for MCV1 in Bangladesh. However, an equally accurate indicator generated in real-time is needed and may be best achieved by replacing EPI record books with electronic records to document a child's vaccination history wherever they get vaccinated. Given the low refusal rate for blood collection in this study, other countries with inaccurate reported or recorded vaccination indicators should consider serological surveillance for measles antibodies as an alternative or corollary to existing surveillance tools. As countries like Bangladesh shift their focus from measles control to elimination and eradication, serological surveillance to estimate population immunity may become an increasingly important indicator [44-46]. A careful consideration of the costs and logistical challenges of serosurveillance need to be weighed against the potential benefits of using immune markers to monitor immunization systems.

\section{Additional file}

Additional file 1. Supplementary tables and figures.

\section{Abbreviations}

Cl: Confidence interval; DHS: Demographic and Health Surveys;

DSS: Demographic Surveillance System; ELIS: Enzyme linked immunosorbent assay; EPI: Expanded Programme on Immunisation; IgG: Immunoglobulin G; OF: Oral fluid; OR: Odds ratio; MICS: Multiple Indicator Cluster Survey; MCV1: Measles containing vaccine, first dose; SES: Socioeconomic status.

\section{Competing interests}

The authors declare that they have no competing interests.

\section{Authors' contributions}

$\mathrm{KH}$ designed study with input from MS, HAE, DB, WM, and OL. KH and MS managed data collection. HAE managed and WM advised on laboratory procedures. $\mathrm{KH}$ conducted analysis and led manuscript preparation with input from MS, HAE, DB, WM, and OL. All authors read and approved the final manuscript.

\section{Acknowledgments}

This manuscript is based on the Kyla Hayford's PhD dissertation research. The authors are grateful to Sanwarul Bari, Kaisar Mahfuzul, Shams El Arifeen and the data collection team at icddr,b; Samir Saha, Roman Mortuza, and Monirul Hoque Pasha at CHRF; the staff at Kumudini Hospital; and David Featherstone at WHO, Vance Dietz at CDC, and Ruth Karron and David Sack at JHSPH for their support during implementation, analysis and manuscript preparation. The study was funded by the Centers for Disease Control and Prevention (CDC). It was not involved in the design, analysis or publication of the study.

\section{Author details}

${ }^{1}$ Department of International Health, International Vaccine Access Center (IVAC), Johns Hopkins Bloomberg School of Public Health, 855 N. Wolfe Street, Suite 600, Baltimore, MD 21205, USA. ${ }^{2}$ International Centre for Diarrhoeal Disease Research, Bangladesh (icddr,b), Mohakhali, Dhaka 1212, Bangladesh. ${ }^{3}$ Child Health Research Foundation (CHRF), Dhaka Shishu Hospital, Dhaka 1212, Bangladesh. ${ }^{4}$ Department of Epidemiology, Johns Hopkins Bloomberg School of Public Health, 615 N. Wolfe Street, Baltimore, MD 21205, USA. ${ }^{5}$ Department of Population, Family and Reproductive Health, Johns Hopkins Bloomberg School of Public Health, Baltimore, 615 N. Wolfe Street, Baltimore, MD 21205, USA.

Received: 19 February 2013 Accepted: 12 December 2013 Published: 20 December 2013

\section{References}

1. WHO: GIVS: global immunization vision and strategy 2006-2015. Geneva: WHO; 2005.

2. United Nations Development Programme: Millennium Development Goals (MDGs). http://www.undp.org/content/undp/en/home/mdgoverview/. 
3. Bos $E$, Batson A: Using immunization coverage rates for monitoring health sector performance: Measurement and interpretation issues In Health, Nutrition and Population (HNP) Discussion Papers. Edited by Bank W. Washington, DC; 2000.

4. Bolton P, Holt E, Ross A, Hughart N, Guyer B: Estimating vaccination coverage using parental recall, vaccination cards, and medical records. Public Health Rep 1998, 113:521-526.

5. Goldman N, Pebley AR: Health cards, maternal reports and the measurement of immunization coverage: the example of Guatemala. Soc Sci Med 1994, 38:1075-1089.

6. Luman ET, Ryman TK, Sablan M: Estimating vaccination coverage: Validity of household-retained vaccination cards and parental recall. Vaccine 2009, 27:2534-2539.

7. Rodewald L, Maes E, Stevenson J, Lyons B, Stokley S, Szilagyi P: Immunization performance measurement in a changing immunization environment. Pediatrics 1999, 103:889-897.

8. Suarez L, Simpson DM, Smith DR: Errors and correlates in parental recall of child immunizations: effects on vaccination coverage estimates. Pediatrics 1997, 99:E3.

9. Valadez JJ, Weld LH: Maternal recall error of child vaccination status in a developing nation. Am J Public Health 1992, 82:120-122.

10. Murray CJ, Shengelia B, Gupta N, Moussavi S, Tandon A, Thieren M: Validity of reported vaccination coverage in 45 countries. Lancet 2003, 362:1022-1027.

11. Lim SS, Stein DB, Charrow A, Murray CJ: Tracking progress towards universal childhood immunisation and the impact of global initiatives: a systematic analysis of three-dose diphtheria, tetanus, and pertussis immunisation coverage. Lancet 2008, 372:2031-2046.

12. Borgdorff MW, Walker GJ: Estimating vaccination coverage: routine information or sample survey? J Trop Med Hyg 1988, 91:35-42.

13. Zuber PL, Yameogo KR, Yameogo A, Otten MW Jr: Use of administrative data to estimate mass vaccination campaign coverage, Burkina Faso, 1999. J Infect Dis 2003, 187:S86-90.

14. Huhn GD, Brown J, Perea W, Berthe A, Otero H, LiBeau G, Maksha N, Sankoh M, Montgomery S, Marfin A, et al: Vaccination coverage survey versus administrative data in the assessment of mass yellow fever immunization in internally displaced persons-Liberia, 2004. Vaccine 2006, 24:730-737.

15. WHO: WHO Immunization Coverage Cluster Survey - Reference Manual. Geneva, Switzerland: World Health Organization; 2005.

16. Brown J, Monasch R, Bicego G, Burton A, Boerma J: An assessment of the quality of national child immunization coverage estimates in population-based surveys. USAID: Measure Evaluation; 2002.

17. Burton A, Monasch R, Lautenbach B, Gacic-Dobo M, Neill M, Karimov R, Wolfson L, Jones G, Birmingham M: WHO and UNICEF estimates of national infant immunization coverage: methods and processes. Bull World Health Organ 2009, 87:535-541.

18. Tapia MD, Pasetti MF, Cuberos L, Sow SO, Doumbia MN, Bagayogo M, Kotloff KL, Levine MM: Measurement of tetanus antitoxin in oral fluid: a tool to conduct serosurveys. Pediatr Infect Dis J 2006, 25:819-825.

19. Vancelik S, Guraksin A, Ayyildiz A, Beyhun NE: Seroepidemiology of poliovirus antibody among the children in Eastern Turkey. Indian J Med Res 2007, 126:528-533.

20. Ohuma EO, Okiro EA, Bett A, Abwao J, Were S, Samuel D, Vyse A, Gay N, Brown DWG, Nokes DJ: Evaluation of a measles vaccine campaign by oral-fluid surveys in a rural Kenyan district: interpretation of antibody prevalence data using mixture models. Epidemiol Infect 2009, 137(2):227-233.

21. Kremer JR, Muller CP: Evaluation of commercial assay detecting specific immunoglobulin $\mathrm{g}$ in oral fluid for determining measles immunity in vaccinees. Clin Diagn Lab Immunol 2005, 12:668-670.

22. Filmer D, Pritchett $L$ : Estimating wealth effects without expenditure data-or tears: an application to educational enrollments in states of India. Demography 2001, 38:115-132.

23. Vyse AJ, Cohen BJ, Ramsay ME: A comparison of oral fluid collection devices for use in the surveillance of virus diseases in children. Public Health 2001, 115:201-207.

24. Microimmune website. http://www.microimmune.co.uk/new/measles_lgG_cap.htm.

25. Siemens Enzygnost Anti-Measles Virus IgG Manual. http://www.healthcare. siemens.com/infectious-disease-testing/systems/bep-iii-system/assays.

26. National Institute of Population Research and Training (NIPORT) MaA, and ORC Macro: Bangladesh demographic and health survey 2004. Maryland, USA: Dhaka, Bangladesh and Calverton; 2005.
27. Measles-Rubella Surveillance and Outbreak Investigation. http://www.searo. who.int/entity/immunization/documents/9789290223504/en/.

28. Leuridan E, Hens N, Hutse V, leven M, Aerts M, Van Damme P: Early waning of maternal measles antibodies in era of measles elimination: longitudinal study. BMJ 2010, 340:C1626.

29. Halsey NA, Boulos R, Mode F, Andre J, Bowman L, Yaeger RG, Toureau S, Rohde J, Boulos C: Response to measles vaccine in Haitian infants 6 to 12 months old. Influence of maternal antibodies, malnutrition, and concurrent illnesses. N Engl J Med 1985, 313:544-549.

30. EPI: Bangladesh EPI coverage evaluation survey 2009. Dhaka, Bangladesh: Expanded Programme on Immunization; 2009

31. Hayford KT, Al-Emran HM, Moss WJ, Shomik M, Bishai D, Levine O: Validation of an anti-measles virus-specific lgG assay with oral fluid samples for immunization surveillance in Bangladesh. J Virol Methods 2013, 193:512-518.

32. Corsi DJ, Bassani DG, Kumar R, Awasthi S, Jotkar R, Kaur N, Jha P: Gender inequity and age-appropriate immunization coverage in India from 1992 to 2006. BMC Int Health Hum Rights 2009, 9(Suppl 1):S3.

33. Kumar D, Aggarwal A, Gomber S: Immunization status of children admitted to a tertiary-care hospital of north India: reasons for partial immunization or non-immunization. J Health Popul Nutr 2010, 28:300-304.

34. Rahman M, Islam MA, Mahalanabis D: Mothers' knowledge about vaccine preventable diseases and immunization coverage in a population with high rate of illiteracy. J Trop Pediatr 1995, 41:376-378.

35. Sheikh S, Ali A, Zaidi AK, Agha A, Khowaja A, Allana S, Qureshi S, Azam I: Measles susceptibility in children in Karachi Pakistan. Vaccine 2011, 29:3419-3423.

36. Giorgi Rossi P, Faustini A, Spadea T, Perucci CA: Choosing immunisation coverage indicators at the local level. Eur J Epidemiol 2004, 19:979-985.

37. Lessler J, Metcalf CJ, Grais RF, Luquero FJ, Cummings DA, Grenfell BT: Measuring the performance of vaccination programs using crosssectional surveys: a likelihood framework and retrospective analysis. PLoS Med 2011, 8:e1001110.

38. Selimuzzaman $A$, Ullah $M$, Haque M: Accuracy of mothers' report regarding vaccination status of the children in urban Bangladesh. J Teach Assoc RMC, Rajshahi 2008, 21:40-43.

39. Lyratzopoulos G, Aston R, Bailey K, Flitcroft J, Clarke H: Accuracy of routine data on MMR vaccination coverage and validity of parental recall of vaccination. Commun Dis Public Health 2002, 5:305-310.

40. Hadler S, Dietz V, Okwo-Bele JM, Cutts F: Immunization in developing countries. In Vaccines. 5th edition. Edited by Plotkin SA, Orenstein WA, Offit PA. Philadelphia: Saunder Elsevier; 2008:1541-1571.

41. Vainio K, Samdal HH, Anestad G, Wedege E, Skutlaberg DH, Bransdal KT, Mundal R, Aaberge IS: Detection of measles- and mumps-specific lgG antibodies in paired serum and oral fluid samples from Norwegian conscripts. Eur J Clin Microbiol Infect Dis 2008, 27:461-465.

42. Goyal A, Shaikh NJ, Kinikar AA, Wairagkar NS: Oral fluid, a substitute for serum to monitor measles IgG antibody? Indian J Med Microbiol 2009, 27:351-353

43. Nigatu W, Nokes DJ, Enquselassie F, Brown DW, Cohen BJ, Vyse AJ, Cutts FT: Detection of measles specific lgG in oral fluid using an FITC/anti-FITC IgG capture enzyme linked immunosorbent assay (GACELISA). J Virol Methods 1999, 83:135-144.

44. Christie AS, Gay A: The measles initiative: moving toward measles eradication. J Infect Dis 2011, 204:S14-17.

45. Keegan R, Dabbagh A, Strebel PM, Cochi SL: Comparing measles with previous eradication programs: enabling and constraining factors. $J$ Infect Dis 2011, 204:S54-61.

46. Hanvoravongchai P, Mounier-Jack S, Oliveira Cruz V, Balabanova D, Biellik R, Kitaw Y, Koehlmoos T, Loureiro S, Molla M, Nguyen H, et al: Impact of measles elimination activities on immunization services and health systems: findings from six countries. J Infect Dis 2011, 204:S82-89.

doi:10.1186/1471-2458-13-1211

Cite this article as: Hayford et al:: Measles vaccination coverage estimates from surveys, clinic records, and immune markers in oral fluid and blood: a population-based cross-sectional study. BMC Public Health 2013 13:1211. 